\title{
Evaluación del Lodo Rojo Activado como Catalizador Heterogéneo en Procesos de Oxidación Avanzada con Radiación Solar para la Degradación de Aguas Agrias Petroleras.
}

\author{
Evaluation of activated red mud as a \\ heterogeneous catalyst in advanced oxidation \\ processes with solar radiation for the degradation \\ of petroleum sour waters.
}

Presentación: 22/06/2020

Aprobación: 28/09/2020

\author{
Guillermo Centeno-Bordones \\ Centro de Investigaciones en Ambiente, Biología y Química, Facultad de Ciencias y Tecnología, \\ Universidad de Carabobo, Valencia - Venezuela. \\ gcenteno1@uc.edu.ve \\ guillermocenteno15@hotmail.com
}

\section{Ygmar Jiménez}

Centro de Investigaciones en Ambiente, Biología y Química, Facultad de Ciencias y Tecnología, Universidad de Carabobo, Valencia, Venezuela.

ygmarjimenez@gmail.com.

\section{Resumen}

Se estudió el potencial catalítico de los lodos rojos activados en la degradación de efluentes (aguas agrias) provenientes del proceso de mejoramiento de crudos pesados y extrapesados tratados mediante procesos de oxidación avanzada utilizando radicación solar. Se aplicó un diseño de experimentos factorial $2^{2}$ con dos puntos al centro. Se emplearon técnicas analíticas para la evaluación del efluente como: DQO, TPH, Conductividad, pH, concentración de sulfuros, concentración de sulfatos, cloruros, ICP-MS e IC. Para la identificación de compuesto orgánicos se aplicó una microextracción es fase sólida y análisis por GC-MS. Los sólidos se analizaron mediante DRX, FTIR, y LECO carbono/azufre. El POA permitió obtener resultados de la disminución de la DQO en un 88,95\% y de TPH en 100\%, logrando también disminuir la concentración de sulfuro en un 99,99\%. Se concluyó que la reacción se realiza 
vía catálisis heterogénea y que la sorción de carbono en el catalizador es menor al 0,2%.

Palabras Claves: Lodo rojo; aguas agrias petroleras; Procesos de oxidación avanzada; Sulfuro; Sulfato

\begin{abstract}
The catalytic potential of activated red mud in the degradation of effluents (sour waters) was studied from the improvement process of heavy and extra-heavy crudes treated by advanced oxidation processes using solar radiation. A factorial experiment design $2^{2}$ with two points to the center was applied. Analytical techniques were used to the effluent to evaluate parameters such as: COD, TPH, Conductivity, $\mathrm{pH}$, concentration of sulphides, concentration of sulfates, chlorides, ICP-MS and IC. For the identification of organic compounds, a solid phase microextraction was applied and then a GC-MS. The solids were analyzed by XRD, FTIR, and carbon / sulfur LECO. The POA allowed obtaining results in the reduction of COD by $88.95 \%$ and TPH by $100 \%$, also reducing the sulfur concentration by $99.99 \%$. It was concluded that the reaction is carried out for heterogeneous catalysis and that the carbon absorption in the catalyst is less than $0.2 \%$.
\end{abstract}

Keywords : Red mud; petroleum sour water; Advanced oxidation processes; Sulfide; Sulfate

\title{
INTRODUCCIÓN
}

Los efluentes de refinación petrolera como las aguas agrias o amargas, se producen en las diferentes etapas del mejoramiento de crudos pesados y extrapesado y su refinación, donde el vapor de agua es utilizado en diferentes tratamientos de crudos como: craqueo catalítico, coquificación retardada, viscoreducción o hidrotratamiento (Naranjo y Lara, 2013; Noguera et al. 2012). El vapor es condensado en presencia de hidrocarburos, de sulfuro de hidrógeno $\left(\mathrm{H}_{2} \mathrm{~S}\right)$ y amoníaco $\left(\mathrm{NH}_{3}\right)$, por lo que estos compuestos son sorbidos en el agua, generando un problema de envergadura en las plantas de tratamiento de aguas residuales, debido a que contienen compuestos orgánicos azufrados, nitrogenados, cetonas, aromáticos y compuestos alifáticos, haciendo de estas aguas una muestra compleja (Noguera y Lara, 2013). Los mejoradores experimentales de crudos pesados y extrapesados de Petróleos de Venezuela, S.A (PDVSA) generan una gran cantidad de este tipo de agua, la cual no cumple con las condiciones paramétricas nacionales para ser vertida en los efluentes de la empresa y ser tratados en las plantas de aguas residuales convencionales, lo que genera un problema logístico y ambiental para la organización, siendo estas almacenadas mientras se busca un tratamiento efectivo para su disposición final. Para el tratamiento de estas aguas se propone utilizar técnicas de química verde que permiten reducir o eliminar las sustancias peligrosas contenidas en estas aguas, a través de otro residuo o desecho industrial como los lodos rojos que pueden ser utilizados como catalizador fotoquímico.

Los lodos rojos se originan del residuo insoluble de la extracción de alúmina mediante el proceso Bayer. Este desecho industrial, está mayormente constituido por una mezcla de óxidos e hidróxidos de hierro, aluminio, titanio, cuarzo, con cantidades menores de arcillas, y otros minerales (Arteaga 2015., Centeno-Bordones et al. 2020). Según el decreto 2635 de la legislación Venezolana (1998), los lodos rojos son considerados desechos peligrosos, 
tóxicos y corrosivos. El lodo rojo se produce en las industrias básicas de Guayana, como la Corporación Venezolana de Guayana - Bauxilum (CVG-Bauxilum). En Venezuela, desde 1983 al 2000, CVG-Bauxilum tuvo una producción de 14 millones de toneladas de lodos rojos, según Galarraga (2002). Actualmente, se estima que más de 35 millones de toneladas de lodo rojo se encuentran almacenadas en los sistemas lagunares de CVG-Bauxilum. Estos residuos terminan depositados en las lagunas próximas al río Orinoco, en una proporción anual de un millón de toneladas de lodos rojos, un cuarto de millón de toneladas de arena roja y dos millones de metros cúbicos de líquidos cáusticos (denominado licor cáustico), según datos proporcionados por el Centro Nacional de Tecnología Química (2012). Siendo estos desechos de la industria metalúrgica un problema ambiental para el ecosistema del Rio Orinoco.

Bhatnagar et al. (2011), reportan que los lodos rojos se pueden utilizar como catalizador en la oxidación de compuestos orgánicos contaminantes, siendo una alternativa sustentable, económica y favorable para el ambiente. Tratar las aguas industriales con lodo rojo es una tecnología prometedora para la sorción y la degradación de compuestos contaminantes (Li, 2011; Liu et al. 2013). Esto daría valor agregado a un pasivo ambiental, que afecta a ecosistemas completos de la Orinoquia Venezolana. De acuerdo a esto y a las propiedades fisicoquímicas del material, los lodos rojos pueden aplicarse para el tratamiento de aguas contaminadas mediante los procesos de oxidación avanzada.

Los procesos de oxidación avanzada (POA) son técnicas que están basadas en la oxidación química o fotoquímica de contaminantes recalcitrantes, que involucran la generación y uso de especies transitorias poderosas, fundamentalmente el radical hidroxilo $\mathrm{OH}^{\circ}$ especie de gran poder oxidante debido a su elevado potencial óxido-reductor $(2,80 \mathrm{~V})$, superado solo por el flúor (Centeno-Bordones y Jiménez, 2018; Clemente, 2010). Los POA vía reacción química son aquellos que se realizan sin la presencia de radiación como Fenton $\left(\mathrm{Fe}^{+2}\right)$ y FentonLike $\left(\mathrm{Fe}^{+3}\right)$. En los procesos de oxidación avanzada de tipo fotoquímicos, la reacción se lleva a cabo en presencia de radiación UV-Visible dentro de los que se encuentran las reacciones fotoFenton $\left(\mathrm{H}_{2} \mathrm{O}_{2} / \mathrm{Fe}^{2+} / \mathrm{UV}\right)$, fotoFenton-like $\left(\mathrm{H}_{2} \mathrm{O}_{2} / \mathrm{Fe}^{3+} / \mathrm{UV}\right)$ y fotocatálisis $\left(\mathrm{TiO}_{2}\right)$. La radiación también se puede aplicar mediante la exposición a la luz solar, aprovechando así la radiación UV y la visible que proporciona el espectro solar, disminuyendo de esta manera costos asociados a equipos y energía requeridos por las lámparas UV.

Al mismo tiempo los POA fotoquímicos involucran a los fotocatalizadores, que pueden ser definidos como unas sustancias que aceleran las reacciones mediante la acción directa de una fuente de emisión de radiación UV-visible (Garcés-Giraldo et al. 2004). En el caso de los fotocatalizadores, se emplean comúnmente semiconductores (sólidos tipo óxido en suspensión acuosa o gaseosa). Existen múltiples de estos catalizadores fotosensibles tales como: $\mathrm{Al}_{2} \mathrm{O}_{3}, \mathrm{Fe}_{2} \mathrm{O}_{3}, \mathrm{TiO}_{2}$ y $\mathrm{MnO}$ entre otros (Pereira y Oliveira, 2012). La reacción catalítica de los semiconductores involucra la absorción de luz ultravioleta por parte del sólido, realizando una óxido-reducción y generando un par electrón-hueco $\left(\mathrm{e}^{-}-\mathrm{h}^{+}\right)$y la consecutiva formación de radicales oxhidrilo $\left(\mathrm{OH}^{\circ}\right)$ y superóxido $\left(\mathrm{O}_{2}^{\circ}\right)$ para la degradación y mineralización de las sustancias orgánicas presentes en el agua (Centeno-Bordones y Jiménez, 2018).

En esta investigación se estudió el potencial catalítico de los lodos rojos activados para la degradación de efluentes petroleros (aguas agrias), provenientes del proceso de mejoramiento 
de crudo pesado y extrapesado mediante un proceso de oxidación avanzada solar, aprovechando las potencialidades en energía solar que posee el país. El poder de mineralización no selectiva que posee la oxidación avanzada fotoquímica y este material de desecho, se convierten en una posible alternativa para el tratamiento de aguas residuales petroleras.

\section{MATERIALES Y MÉTODOS}

\section{Diseño de experimentos}

La investigación se desarrolló a través de un diseño de experimentos (DDE) del tipo factorial $2^{2}$ con dos puntos al centro, utilizando el proceso de oxidación fotoquímica con una duración de $4 \mathrm{~h}$ de exposición solar, teniendo como variable de respuesta la concentración de la DQO, y como factores de entrada: a.- Cantidad de catalizador (lodo rojo neutralizado con salmuera y calcinado LRS400), b.- Cantidad de peróxido de hidrógeno al 30\% (grado analítico marca Eka Nobel). En la Tabla 1 se observan los factores de entrada, los niveles y las codificaciones que toman las variables en el DDE. Los datos experimentales fueron analizados con el programa estadístico Statgraphic Centurion XVI, versión libre. Los cálculos realizados mediante éste programa se hicieron utilizando un nivel de confianza del 95\%.

\begin{tabular}{|c|c|c|}
\hline FACTORES & NIVELES Y CODIFICACIÓN & VARIABLE DE RESPUESTA \\
\hline Cantidad de catalizador & $6 \mathrm{~g} / \mathrm{L}(-1)$ & \\
(LRS400) & $7 \mathrm{~g} / \mathrm{L}(0)$ & \\
& $8 \mathrm{~g} / \mathrm{L}(1)$ & Demanda Química de \\
& $66,66 \mathrm{~g} / \mathrm{L}(-1)$ & \\
Peróxidoeno (DQO) & $80 \mathrm{~g} / \mathrm{L}(0)$ & \\
$30 \%$ & $100 \mathrm{~g} / \mathrm{L}(1)$ & \\
\end{tabular}

Tabla 1: Factores, niveles y codificación del diseño de experimento factorial 22 con dos puntos al centro para la fotodegradación de agua agria.

\section{Reacción de Oxidación Avanzada}

El proceso de oxidación avanzada se llevó a cabo en un balón de tres bocas de volumen $250 \mathrm{~mL}$ (vidrio borosilicato), conectado a un embudo de adición para la dosificación de peróxido de hidrógeno al 30\% según la metodología utilizada por Yamal et al. (2013), un tubo de enfriamiento para el control de vapores y un termómetro para el seguimiento de la temperatura de reacción, manteniendo una agitación constante a través de un agitador magnético (SLHS-SI Analytics), el montaje experimental se desarrolló según la configuración propuesta por Ramos, y Jiménez (2016) (Figura 1). Al término de las reacciones, se filtró la mezcla final para separar el catalizador, y luego se neutralizó utilizando hidróxido de sodio al 20\% (Sigma Aldrich). El tiempo de la reacción fue de $4 \mathrm{~h}$, considerando el intervalo de tiempo del cenit solar en las siguientes coordenadas Norte $10^{\circ} 22^{\prime} 03,16^{\prime \prime}$ y Este $67^{\circ} 02^{\prime} 38,25^{\prime \prime}$ a 1316 msnm, Los Teques, Estado Miranda-Venezuela. La medición de la radiación solar (Wh/ $\mathrm{m}^{2}$ ) se realizó mediante un piromanómetro ISO First Class marca Climatronics Corporation. 


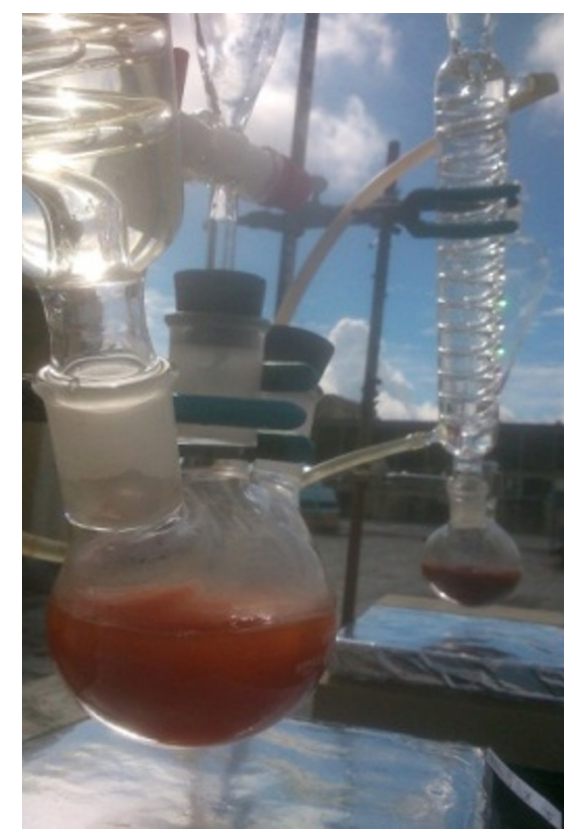

Fig.1: Configuración del montaje experimental para el estudio del proceso de oxidación avanzada utilizando radiación solar y lodos rojos tratados como catalizador.

\section{Caracterización post-reacción}

El proceso de caracterización del agua tratada se inició midiendo el pH a través de un pHmetro digital Orión 330 Thermo. La conductividad se midió mediante un multiparametro WTW Tetracon 325 Cond 197i. La Demanda Química de Oxígeno (DQO) se realizó mediante el método HACH DR2010 y la de concentración de Hidrocarburos Totales de Petróleo (TPH) a través de la técnica de fluorescencia ultravioleta (UVF) Sitelab:EDRO:16 descrita por la EPA 8015,2000. La concentración de cloruros se midió a través de la técnica argentométrica según el Standard Methods 4500 Cl-B, (1995). La determinación de metales se realizó mediante la técnica de Espectroscopia de Plasma Inducido acoplado a Masa (ICP-MS) según el Método EPA 6020B (revisión 2, Julio 2014) para ello se utilizó un ICP-MS Agilent Technologies 7500ce con las siguientes características: Frecuencia: 27,12 MHz Potencia RF: máximo 1600 W Antorcha: tipo Fassel.

La concentración de sulfuros en la muestra de aguas agrias se midió utilizando la técnica de estandarización del Na2S marca Avantor VWR al 98\%, y se realizó bajo la norma ASTM D 4658-03. Se empleó un electrodo selectivo a iones sulfuro (Thermo Scientific Orion silver/sulfide electrode), este se conectó a un $\mathrm{pH}$-metro con escala expandible a $\mathrm{mV}$ (Thermo Scientific Orion, 5-Star). La determinación de sulfatos en el agua agria se realizó mediante el método HACH 8051-SulfaVer4-Method USEPA 375.4. En el agua agria fototratada se midió la concentración de sulfatos y se identificaron los iones generados para los mejores resultados mediante un Cromatógrafo Iónico (IC) Professional 850 de Metrohm con sistema de ultrafiltración, y un detector de conductividad en supresión química secuencial con detector amperométrico de pulsos (PAD). 
La determinación de las especies orgánicas se realizó mediante cromatografía de gases acoplada a masas (GC-MS) para especies orgánicas complejas, por lo que se requirió de un tratamiento previo de la muestra para eliminar la fase acuosa, y para ello se aplicó una microextracción en fase sólida mediante una pequeña columna C-18 (de polidimetilsiloxano) marca SUPELCO SPME, usándose como agente de activación de la columna Metanol (Merk) y como solvente diclorometano (Sigma Aldrich). La GC-MS se basó en el método ASTM D5790-95 (2012), ensayo normalizado para medidas de compuestos orgánicos purgables en agua por columna capilar de cromatografía de gases/espectrometría de masa. Se empleó un cromatógrafo de gases: marca Agilent Technologies modelo 6890N.

Caracterización del catalizador gastado y sólidos generados

La caracterización del catalizador gastado se realizó mediante difracción de rayos $\mathrm{X}$ (DRX), con un difractómetro PANalytical modelo X` pert PRO. La espectroscopia infrarroja con transformada de Fourier (FTIR) se llevó a cabo mediante un equipo FTIR Spectrometer Frontier, Perkin-Elmer. La determinación de Carbono elemental se realizó con un equipo LECO serie CS844 carbono/ azufre analizador.

\section{RESULTADOS Y DISCUSIÓN}

El diseño experimental permitió obtener 6 corridas que se reflejan en la Tabla 2, aplicándose para éste modelo sólo los procesos de oxidación avanzada de tipo fotoquímicos. En dicha Tabla se aprecian los datos obtenidos del DDE. Se observa que la variación del pH sufre una disminución por debajo del ajuste realizado con ácido sulfúrico; la oxidación de especies orgánicas disminuye también el $\mathrm{pH}$. Por otra parte se presenta un proceso no fotoquímico Fenton-Like con las condiciones de la corrida 4, y la repetición de la corrida 4.

\section{Reacción de Oxidación Avanzada y su efecto en el efluente}

En la Tabla 2 se observa que la cantidad de DQO inicial en el agua agria fue de $18100 \mathrm{mg} / \mathrm{L}$ y mediante la aplicación de las técnicas fotoquímicas se logró reducir hasta un mínimo de $2000 \mathrm{mg} / \mathrm{L}$, lo cual representa una disminución de un 88,9\% con respecto al valor inicial, lo que evidencia la eficiencia de la aplicación de los procesos de oxidación avanzada solar utilizando como catalizador el lodo rojo. Éste efecto catalítico se puede observar con la disminución de la concentración de hidrocarburos totales de petróleo presentes en la muestra, lográndose reducir la concentración a $0,00 \mathrm{mg} / \mathrm{L}$ teniendo una efectividad de la fotoxidación en $100 \%$. Es importante mencionar, que en los procesos de oxidación avanzada de tipo solar utilizando lodo rojo neutralizado con salmuera y calcinado (LRS400) como catalizador, se aplican dos técnicas fotoquímicas consecutivas como fotoFenton-like y fotocatálisis con semiconductores como el óxido de titanio y óxido de manganeso (Centeno-Bordones y Jiménez, 2018). El lodo rojo se presenta como un catalizador sinérgico del cual se extrae todo su potencial catalítico evidenciado en los resultados obtenidos en los diseños de experimentos aplicados. Este material de desecho de la industria metalúrgica puede ser recuperado y activado para aplicaciones catalíticas en los procesos de oxidación avanzada, con un éxito importante en la degradación y mineralización de aguas agrias con alto contenido de DQO. 


\begin{tabular}{|c|c|c|c|c|c|c|c|c|c|}
\hline $\begin{array}{c}\text { Corridas } \\
\mathbf{y} \\
\text { códigos }\end{array}$ & $\begin{array}{c}\mathrm{pH} \\
\text { Inicial } \\
\pm 0,01\end{array}$ & $\begin{array}{c}\mathrm{pH} \\
\text { final } \\
\pm 0,01\end{array}$ & $\begin{array}{c}\text { Condct } \\
\mu \mathrm{S} / \mathrm{Cm} \\
\pm 0,1\end{array}$ & $\begin{array}{c}\text { [H2O2] } \\
\mathbf{g} / \mathrm{L} \\
\pm 0,01\end{array}$ & $\begin{array}{c}\text { DQO } \\
\pm 1,00 \\
\mathrm{ppm}\end{array}$ & $\begin{array}{c}\text { Reducción } \\
\text { de la } \\
\text { DQO } \\
\pm 0,01 \\
\%\end{array}$ & $\begin{array}{c}\text { TPH } \\
\pm 0,001 \\
\mathrm{ppm}\end{array}$ & $\begin{array}{c}\text { Reducción } \\
\text { de los } \\
\text { TPH } \\
\pm 0,01 \\
\%\end{array}$ & $\begin{array}{c}\text { Relación } \\
\text { DQO } \\
:\left[\mathrm{H}_{2} \mathrm{O}_{2}\right]\end{array}$ \\
\hline Agua agria & 10,5 & - & 2,80 & - & 18100 & - & 99,99 & - & - \\
\hline $1(-1,-1)$ & 2,05 & 1,60 & 62,9 & 17,60 & 2300 & 87,29 & 0,040 & 99,95 & $1: 0,97$ \\
\hline $2(-1,1)$ & 2,08 & 1,59 & 59,3 & 22,01 & 2500 & 86,18 & 0,080 & 99,91 & $1: 1,21$ \\
\hline $3(1,-1)$ & 2,00 & 1,66 & 64,3 & 14,67 & 2300 & 87,29 & 0,000 & 100,00 & $1: 0,81$ \\
\hline $4(1,1)$ & 1,99 & 1,60 & 64,5 & 18,99 & 2000 & 88,95 & 0,000 & 100,00 & $1: 1,04$ \\
\hline $5(0,0)$ & 2,08 & 1,62 & 63,7 & 17,69 & 2300 & 87,29 & 0,000 & 100,00 & $1: 0,97$ \\
\hline $6(0,0)$ & 2,06 & 1,60 & 63,8 & 17,51 & 2200 & 86,18 & 0,000 & 100,00 & $1: 0,96$ \\
\hline Fenton-Like & 2,04 & 2,50 & 32,4 & 38,87 & 3500 & 80,66 & 0,078 & 99,92 & $1: 2,14$ \\
\hline 4 & 2,03 & 1,73 & 64,7 & 18,76 & 1900 & 89,50 & 0,000 & 100,00 & $1: 1,2$ \\
\hline Rep (4) & 2,0 & & & & & & & \\
\hline
\end{tabular}

Tabla 2: Diseño de Experimento factorial $2^{2}$ con dos puntos al centro para la degradación del agua agria en POA con lodo rojo como catalizador utilizando radiación solar.

La reacción no fotoquímica se llevó a cabo para determinar el efecto de la radiación solar y cómo este factor influye en el proceso de oxidación, en la reacción Fenton-Like bajo las condiciones de la corrida 4. Se obtuvo la degradación del 80,66 \% de la DQO y 99,92\% de la $\mathrm{TPH}$, resultando que el proceso de oxidación de tipo no fotoquímico es igualmente efectivo en la degradación y mineralización de la materia orgánica e inorgánica presente en agua agria petrolera, logrando mejores resultados que los obtenidos por Rubio-Clemente et al. (2014) utilizando un catalizador comercial.

Cuando se analizan las relaciones de DQO: $\mathrm{H}_{2} \mathrm{O}_{2}$ aplicadas en el diseño, se puede observar que en la corrida 4 dicha relación es inicialmente de 1:5,5 lo cual corresponde con lo expresado por Guimarães et al. (2012) en un estudio de aguas agrias brasileñas. En las relaciones DQO: $\mathrm{H}_{2} \mathrm{O}_{2}$ remanentes se puede ver (en la última columna de la Tabla 2) que la cantidad de peróxido de hidrógeno en las reacciones es baja, debido al consumo de éste reactivo durante el inicio de la reacción, coincidiendo con la etapa de mayor producción de radicales libres por la activación del catalizador y la fotólisis solar del peróxido de hidrógeno (Marquez,2015).

El montaje experimental permitió obtener valores bajos para la DQO y TPH, lo cual implica que el diseño de experimento aplicado tuvo su mejor resultado en la corrida 4 (Tabla 2). Esto se debe a que la aplicación de los procesos de oxidación avanzada solar, utilizando como catalizador el lodo rojo tiene efectos importantes en la reducción de la materia orgánica contenida en la muestra. Según Jang et al. (2007) el ajuste de $\mathrm{pH}$ juega un papel importante en la especiación de la hematita, permitiendo que esta especie mediante la hidratación y dilución forme un complejo de metal-carga-ligando permitiendo dar lugar a la reacción fotoFenton-like (Centeno-Bordones y Jiménez, 2018), logrando que se forme una especie compleja que tiene una absorbancia en el espectro electromagnético visible alrededor de $580 \mathrm{~nm}$ (Ramos, y Jiménez, 2016). 
Adicionalmente, el lodo rojo neutralizado con salmuera y calcinado a $400{ }^{\circ} \mathrm{C}$ permite que las especies cristalinas presentes, como semiconductores, lleven a cabo la formación de radicales libres mediante la generación del par electrón-hueco a través de la absorción de radiación UV solar entre 300-400 $\mathrm{nm}$ para la anatasa, y 260-290 nm para la hematita, ocurriendo así la óxido-reducción del peróxido de hidrógeno en medio acuoso (Garcés-Giraldo et al. 2004 ; Centeno-Bordones y Jiménez, 2018). Esta diversidad de especies fotosensibles que absorben a diferentes longitudes de ondas del espectro electromagnético, es el motivo por el cual se realizaron las reacciones utilizando radiación solar. La radiación solar en la zonas de los Altos Mirandinos (1316 msnm) fue de $1932 \mathrm{~W} / \mathrm{m}^{2}$, lo que implica que la cantidad de energía en forma de radiación en la zona este estimada en 5,1 Kw/m2día. Representando estos datos las excelentes condiciones para el aprovechamiento de energía solar como fuente alternativa en aplicaciones fotoquímicas.

\section{Análisis del Diseño de experimento}

Desde el punto de vista del análisis estadístico, el R-Cuadrado es de 95,625 \%, explicando en esta proporción la variabilidad de los datos de la DQO. El estadístico R-cuadrado ajustado, que es adecuado para comparar modelos con diferente número de variables independientes, es de 89,062 \% por lo que se puede decir que el diseño de experimento se ajusta en un $89 \%$ al modelo matemático factorial $2^{2}$ con dos puntos al centro. En este caso, dos efectos tienen una valor-P menor que 0,05 indicando que son significativamente diferentes de cero con un nivel de confianza del 95,0 \% (en Figura 2, se observa que dos barras sobrepasan la línea de significancia). Para este análisis estadístico se determinaron los supuestos de la ANOVA como la normalidad mediante el test de Shapiro-Wilk, arrojando que no se puede rechazar la idea que los datos describen una distribución normal con un $95 \%$ de confianza. La independencia se midió mediante el test de Durbin-Watson afirmando que no se puede rechazar la idea que existe correlación de los datos. La prueba de varianza constante se aplicó mediante el método gráfico predichos versus residuos y no se observó patrón de puntos en la gráfica. Por lo que el modelo estadístico aplicado cumple con los supuestos de la ANOVA.

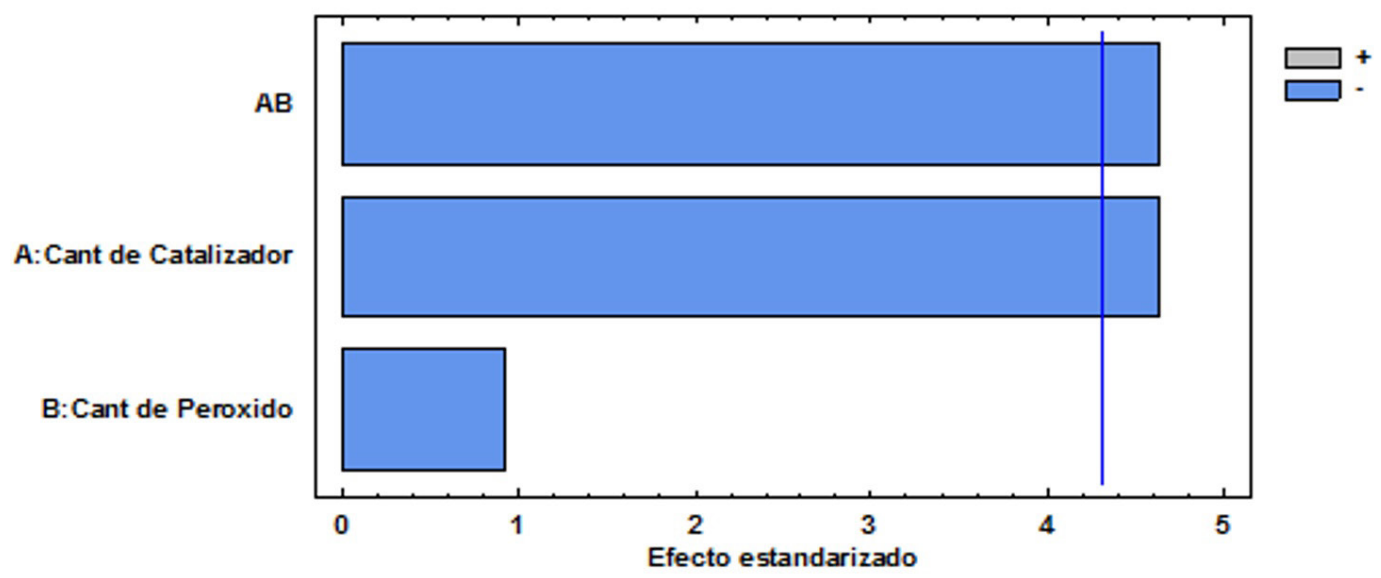

Fig. 2: Diagrama de Pareto estandarizado para DQO para el DDE.

En la Figura 2 se observa el diagrama de Pareto para el análisis de varianza (ANOVA) 
aplicado al DDE. El ANOVA prueba la significancia estadística de cada efecto comparando su cuadrado medio contra un estimado del error experimental (Gutiérrez y De la Vara, 2008). En el diagrama se pueden observar las variables de entrada escogidas: cantidad de catalizador (A) y cantidad de peróxido de hidrógeno (B), bajo condiciones experimentales codificadas en la Tabla 2. Luego de someter los datos al análisis estadístico ANOVA (Figura 2), se obtuvo que el efecto doble de las variables es significativo debido a que sobrepasa la línea de significancia estadística, por lo que se puede decir que ambas factores influyen en la variable de respuesta (DQO). También se puede observar que la cantidad de catalizador es significativa y tiene efecto directo sobre la variable de respuesta, caso que no ocurre con la cantidad de peróxido de hidrógeno, para este diseño experimental.

En la Figura 3 se puede ver la superficie de respuesta estimada de la variable de la DQO, a partir de los datos obtenidos de la Tabla 2. Se observa como resultado una curvatura de la superficie típica de los DDE con puntos al centro, resultando la mejor región experimental el vértice más bajo de la red. Para la variable cantidad de peróxido, una mayor cantidad (código 1) es el valor de la mejor región de reducción para la respuesta de la DQO, al igual que para la variable cantidad de catalizador. Lo anterior permite sugerir, que los valores tomados por estos códigos experimentales $(1,1)$, son los mejores niveles para la disminución significativa de la variable de respuesta analizada.

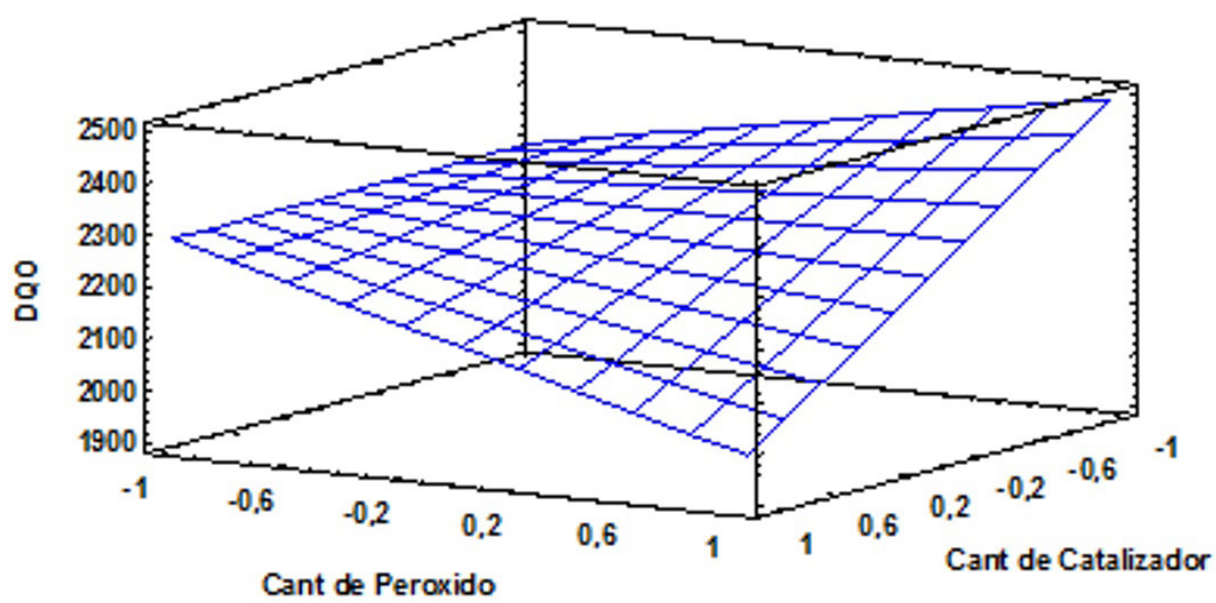

Fig. 3: Superficie de respuesta estimada DQO para el DDE.

\section{Metales y especies de azufre presentes en el agua agria fototratada}

En la Tabla 3 se observa la cantidad de metales presentes que fueron determinados mediante ICP en el agua agria tratada mediante POA solar, donde se observó que la concentración de los metales de transición como el níquel, vanadio, molibdeno y cobre es de $1 \mathrm{ppm}$. Adicionalmente se observaron metales característicos asociados al petróleo venezolano de la faja petrolífera del Orinoco (FPO) como el vanadio, manganeso e hierro (González y Núñez, 2008). También se determinó que existe una cantidad de metales alcalinos y alcalinotérreos típicos de la litología de la zona, y de la composición de sales presentes en las aguas 
connatas como el potasio, sodio, calcio y magnesio, tal como demostró Castro et al. (2019). La cantidad de metales presentes en los lodos rojos también realizarán su aporte en el agua tratada tal como lo afirma Centeno-Bordones et al. (2020). Entre ellos están el sodio, potasio, calcio, hierro, manganeso entre otros, lo cual pudiese explicar el incremento de algunos elementos en el agua agria tratada.

\begin{tabular}{|c|c|c|c|c|c|c|c|c|c|}
\hline \multicolumn{8}{|c|}{ Determinación de Metales } & \multicolumn{2}{|c|}{$\begin{array}{l}\text { Determinación de } \\
\text { especies de azufre }\end{array}$} \\
\hline $\begin{array}{l}\text { Muestra de } \\
\text { efluente }\end{array}$ & $\begin{array}{c}\text { Ni,v, } \\
\text { Mo,Cu } \\
\pm 0,05 \\
\mathrm{mg} / \mathrm{L}\end{array}$ & $\begin{array}{c}\mathrm{Na} \\
\pm 0,05 \\
\mathrm{mg} / \mathrm{L}\end{array}$ & $\begin{array}{c}K \\
\pm 0,05 \\
\mathrm{mg} / \mathrm{L}\end{array}$ & $\begin{array}{c}\text { Mg } \\
\pm 0,05 \\
\mathrm{mg} / \mathrm{L}\end{array}$ & $\begin{array}{c}\mathrm{Ca} \\
\pm 0,05 \\
\mathrm{mg} / \mathrm{L}\end{array}$ & $\begin{array}{c}F e \\
\pm 0,05 \\
\mathrm{mg} / \mathrm{L}\end{array}$ & $\begin{array}{c}\text { Mn } \\
\pm 0,05 \\
\mathrm{mg} / \mathrm{L}\end{array}$ & $\begin{array}{c}S^{-2} \\
\pm 0,1 \\
m g / L\end{array}$ & $\begin{array}{c}\mathbf{S O}_{4}^{-2} \\
\pm 0,31 \%\end{array}$ \\
\hline Agua agria & 1 & 142 & 512 & 3,96 & 5,26 & 1 & 1 & 24000 & 1,19 \\
\hline $\mathrm{FQ}(4)$ & 1 & 311 & 540 & 4,77 & 120 & 56 & 45 & $<1$ & 3,69 \\
\hline Fenton-Like 4 & 1 & 149 & 594 & 3,99 & 72,80 & 63 & 55,20 & $<1$ & 2,04 \\
\hline Rep FQ4 & 1 & 309 & 539 & 4,85 & 118 & 57 & 44 & $<1$ & 3,69 \\
\hline
\end{tabular}

Tabla 3: Metales y especies de azufre presentes en las aguas agrias tratadas.

El incremento en la cantidad de metales en el efluente tratado es debido a la lixiviación del hierro y el manganeso por la disminución del $\mathrm{pH}(1,70)$ en la reacción, donde la concentración de hierro pasa de 1 ppm a 56 ppm, tal y como se observa en la Tabla 3. La lixiviación de las especies antes mencionadas, incrementa la concentración a $56 \mathrm{ppm}$ para el hierro y 45 para el manganeso. En la réplica del experimento los valores de lixiviación de estos metales fueron cercanos a los obtenidos en la reacción fotoquímica bajo las condiciones de la corrida 4 (FQ4). De la misma manera se tiene la comparación con la reacción Fenton-Like, en este proceso la lixiviación del hierro y el manganeso es mayor, obteniendo valores por encima a la de los procesos fotoquímicos. Esto es debido a que en los procesos fotoquímicos ocurre un efecto protector de especies complejas metal-peróxido, impidiendo la lixiviación de metales en condiciones de irradiación (Martínez et al. 2005; Ramos, 2017).

En la Tabla 3 se pueden observar las especies de azufre presentes en el agua agria y en los respectivos tratamientos, evidenciándose que la concentración de sulfatos se incrementa considerablemente y la concentración de sulfuros disminuye luego del proceso de oxidación avanzada. El petróleo de la FPO contiene azufre como especie asociada a su estructura orgánica, debido al contenido de éste compuesto en la fase inorgánica del yacimiento (roca) (Alboudwarej et al. 2006). Después del proceso de fotoxidación la reacción permitió llevar el sulfuro hasta sulfato en un 99,99\%. Esto pudo haber ocurrido directamente por acción catalítica del manganeso o por sorción de sulfuro por parte del hierro $\left(\mathrm{Fe}^{+3}\right)$ presente en el catalizador. Esta reacción catalítica se lleva acabo debido al contenido de manganeso en el lodo rojo, que al ajustar el $\mathrm{pH}$ con ácido sulfúrico, permite la reacción con este, para formar sulfato de manganeso, una especie promotora altamente probada para la oxidación de sulfuros en la industria minera (Salas, 2005). Por su parte la presencia de peróxido de hidrógeno en el medio proporciona un alto contenido de oxígeno que también permite llevar a cabo la reacción de oxidación inorgánica del azufre (Ecuación 1). El aporte de la fase orgánica al contenido de sulfatos se debe a que el azufre se encuentra enlazado a la matriz del hidrocarburo, bajo la forma de sulfuros, disulfuros, tioles y tiofenos simples y complejos, que son oxidados bajo estas condiciones experimentales (Castro et al. 2019). 


$$
S^{-2}+2 \mathrm{O}_{2} \frac{a \cdot-\mathrm{MnSO}_{4}}{b \cdot-\mathrm{H}_{2} \mathrm{O}_{2}} \rightarrow \mathrm{SO}_{4}^{-2}
$$

\section{El lodo rojo activado como catalizador heterogéneo en los POA}

En la Tabla 4, se observan los resultados obtenidos específicamente en la corrida 4 (FQ4), donde se pudo obtener un $\mathrm{pH}$ de 1,70 con una disminución de la concentración de la DQO en un $88,95 \%$ y de la concentración de hidrocarburos totales de petróleo del 100\%. Para esta corrida la concentración de peróxido remanente es de 18,99 g/L y la lixiviación del hierro en esta corrida experimental fue de 3,11\%. En la reacción Fenton- like se observa que la degradación de la DQO y los TPH es menor que en los procesos fotoquímicos, permitiendo este proceso una menor generación de lixiviado, al mismo que se observa una mayor concentración de peróxido remanente en el efluente tratado.

\begin{tabular}{|c|c|c|c|c|c|c|c|}
\hline Muestra & $\begin{array}{c}\text { pH } \\
\text { final } \\
\pm 0,01\end{array}$ & $\begin{array}{l}\text { DQO } \\
\pm 1,00 \\
\text { ppm }\end{array}$ & $\begin{array}{c}{[\mathrm{H} 2 \mathrm{O} 2]} \\
\pm 0,01 \\
\mathrm{~g} / \mathrm{L}\end{array}$ & $\begin{array}{c}\text { DQO } \\
\pm 0,01 \\
\%\end{array}$ & $\begin{array}{c}\text { TPH } \\
\pm 0,001 \\
\text { ppm }\end{array}$ & $\begin{array}{c}\text { TPH } \\
\pm 0,01 \\
\%\end{array}$ & $\begin{array}{c}\text { Hierro Lixiviado } \\
\%\end{array}$ \\
\hline Agua agria & - & 18100 & - & - & 99,000 & - & - \\
\hline FQ4 & 1,70 & 2000 & 18,99 & 88,95 & 0,000 & 100,00 & 3,11 \\
\hline Fenton-Like4 & 2,50 & 3500 & 38,87 & 80,66 & 0,079 & 99,92 & 3,77 \\
\hline Rep FQ4 & 1,73 & 1900 & 17,68 & 89,50 & 0,000 & 100,00 & 3,16 \\
\hline
\end{tabular}

Tabla 4: Valores para el mejor resultado del diseño de experimento aplicado con lixiviado de hierro, replica y Fenton-like.

Cuando se realiza la réplica de esta corrida (Rep FQ4) se obtienen valores similares a los obtenidos inicialmente con un reducción de la concentración de la DQO en 89,50\% y de la TPH en $100 \%$, demostrando que los resultados obtenidos tienen reproducibilidad, y esta propiedad también se obtuvo en los valores de lixiviación de hierro en la fotoreacción con un 3,16 \%, resultando valores bajos de lixiviación de hierro. Lo anteriormente se explica debido al efecto protector por la formación de especies complejas entre el metal-peróxido de hidrogeno según Ramos (2017), Martínez et al. (2004) y Ríos-Enríquez et al. (2003). Los investigadores afirman que a mayor concentración de peróxido de hidrogeno se obtiene un menor lixiviado de hierro, ya que las especies complejas hierro-hidroperoxil previenen la lixiviación fotoinducida por la radiación UV-visible. Este análisis estaría sustentado por Martínez et al. (2004) y Ramos (2017) mediante las ecuaciones de la (2) hasta la (5):

$$
\begin{aligned}
& \equiv \mathrm{Fe}^{+3}-\mathrm{OH}+\mathrm{H}_{2} \mathrm{O}_{2} \leftrightarrow\left(\mathrm{H}_{2} \mathrm{O}_{2}\right) \mathrm{s} \\
& \left(\mathrm{H}_{2} \mathrm{O}_{2}\right) \mathrm{s} \leftrightarrow\left(\equiv \mathrm{Fe}^{+2}-{ }^{\circ} \mathrm{O}_{2} \mathrm{H}\right)+\mathrm{H}_{2} \mathrm{O} \\
& \left(\equiv \mathrm{Fe}^{+2}-{ }^{\circ} \mathrm{O}_{2} \mathrm{H}\right) \leftrightarrow \equiv \mathrm{Fe}^{+2}+\mathrm{HO}_{2}{ }^{\circ} \\
& \equiv \mathrm{Fe}^{+2}+\mathrm{H}_{2} \mathrm{O}_{2} \rightarrow \equiv \mathrm{Fe}^{+3}-\mathrm{OH}+\mathrm{HO}^{\circ}
\end{aligned}
$$


En las ecuaciones 2 a la 5, se puede apreciar que en las reacciones llevadas a cabo en presencia de $\mathrm{Fe}^{+3}$ y peróxido de hidrógeno, la lixiviación de hierro muestra una tendencia decreciente cuando aumenta la concentración de oxidante (Martínez et al. 2004).

Este comportamiento de menor lixiviación de metales para condiciones oxidantes más fuertes parece ser diferente al que muestra el Fenton- like heterogéneo, que en reacciones de condiciones semejantes a las reacciones fotoquímicas en el que se observó una mayor lixiviación a altas concentraciones de peróxido (Martínez et al. 2004). La descomposición catalítica del peróxido de hidrógeno sobre los óxidos de hierro se basa en la formación de especies complejas de peróxido con sitios activos de $\mathrm{Fe}^{+3}$ en el superficie del catalizador después de una serie de reacciones en las que este complejo de superficie metálica sufre diferentes estados electrónicamente excitados que permiten la disociación en el radical peróxido, (Lin y Gurol, 1998).

Según la Ecuación 5, los sitios reducidos de hierro pueden reaccionar con peróxido de hidrógeno para regenerar los sitios de hierro oxidados nuevamente. A partir de este mecanismo, la disminución general de la lixiviación de hierro para altas concentraciones de oxidantes podría ser atribuido al efecto de blindaje del metal circundante formando especies complejas de $\mathrm{H}_{2} \mathrm{O}_{2}$ que evitan la lixiviación fotoinducida fenómeno por irradiación UV-vis. Los autores Ríos- Enríquez et al. (2003) también han propuesto la hipótesis de lixiviación de $\mathrm{Fe}^{+2}$ a partir de catalizadores que contienen $\mathrm{Fe}^{+3}$ como resultado de la reducción fotoquímica de las especies de $\mathrm{Fe}^{+3}$ acumulado en la fase acuosa a menos que se vuelve a oxidar por un exceso de peróxido de hidrógeno.

\section{Sorción de carbono elemental del lodo rojo activado}

En la Tabla 5 se observan las concentraciones de carbono elemental que contiene el catalizador, que consiste en lodo rojo neutralizado y calcinado (LRS400) empleado para los procesos de oxidación avanzada utilizando radiación solar, Este material de desecho de la industria metalúrgica activado tiene la capacidad de comportarse como un catalizador con actividad fotoquímica y fotocatalítica debido a que contiene especies de óxido férrico, óxido de titanio y óxido de manganeso que tienen comprobada actividad catalítica (CentenoBordones et al. 2020). Según autores como Bathnagar et al. (2011), el material descrito puede comportarse como un sorbente de metales pesados, compuestos orgánicos e inorgánicos. En la Tabla 5 se aprecia el contenido de carbono elemental en el catalizador de lodo rojo activado (LRS400), arrojando valores que confirman la presencia de carbono con una concentración de 0,433 \% en peso. La presencia de carbonos puede deberse a la cantidad de carbonato presente en la cancrinita de tipo sodalita en el catalizador LRS400 determinada mediante el análisis de difracción de rayos $\mathrm{X}$ del material reportado por Centeno-Bordones et al. (2020).

\begin{tabular}{|c|c|c|c|}
\hline $\begin{array}{c}\text { Muestra de } \\
\text { Catalizador }\end{array}$ & $\begin{array}{c}\text { Carbono } \\
\text { elemental } \\
\pm 0,002 \mathrm{ppm}\end{array}$ & $\begin{array}{c}\text { Carbono sorbida } \\
\pm 0,002 \mathrm{ppm}\end{array}$ & $\begin{array}{c}\text { DQO por sorción de carbono } \\
\pm 0,001 \%\end{array}$ \\
\hline LRS400 & 0,433 & - & - \\
\hline FQ4 & 0,985 & 33,12 & 0,132 \\
\hline Fenton-like4 & 0,995 & 33,72 & 0,186 \\
\hline Rep FQ4 & 0,859 & 25,56 & 0,141 \\
\hline
\end{tabular}

Tabla 5: Determinación de la concentración de carbono elemental en el catalizador lodo rojo LRS 400 antes de la reacción y gastado en el proceso de oxidación. 
En el catalizador gastado en la corrida numero 4 (FQ4) se puede apreciar cómo se eleva la concentración de carbono hasta 0,985 ppm en el catalizador usado, elevando su concentración de carbono sorbida en el catalizador hasta 33,12 ppm, representando este valor una reducción de $0,182 \%$ en la DQO. Cuando se realiza la repetición de esta determinación se evidencia que la sorción de carbono en el catalizador fue de 25,56 ppm representando una reducción de la $\mathrm{DQO}$ de $0,141 \%$. El proceso de oxidación avanzada de tipo no fotoquímico como el Fenton-like, permitió una sorción de carbono de 33,72 ppm, siendo este el proceso que facilitó la mayor sorción de este elemento en el material. Sin embargo el aporte en la reducción de la DQO del agua agria por esta vía fue de 0,186 \%. Estos datos evidencian que la neutralización y la activación térmica del lodo rojo permiten funcionalizar el material potenciando su capacidad catalítica debido a la reconfiguración de especies activas en la superficie del catalizador y a la formación de complejos fotoactivos que permiten la generación de radicales en el medio(Gupta et al. 2003 ; Folgoso, 2015) .

Especies generadas en el proceso de oxidación avanzada aplicado a las aguas agrias petroleras

En la Tabla 6 se pudo identificar las especies orgánicas resultantes de la reacción Fentonlike, encontrándose fenol y alcoholes debido a la oxidación incompleta de las especies presentes en el agua agria (Montes, 2014), debido a que en las reacciones Fenton-like la generación de radicales libres es menor que en los procesos fotoquímicos. También se puede observar en la Tabla la identificación del azufre elemental formado a partir del sulfuro férrico en medio ácido, debido posiblemente a la sorción del azufre en el catalizador, lo cual se ve favorecido por las condiciones del medio para la generación de sulfato ferroso y azufre elemental (Hinojosa, 2002).

\begin{tabular}{|c|c|c|c|c|}
\hline $\begin{array}{c}\text { Procedencia de la } \\
\text { muestra }\end{array}$ & Grupo Funcional & Compuestos & $\begin{array}{c}\text { Tiempo de } \\
\text { Retención } \\
(\mathbf{m i n})\end{array}$ & $\begin{array}{c}\text { Coincidencia } \\
\text { Espectral }\end{array}$ \\
\hline \multirow{5}{*}{ Fenton-Like4 } & Aromáticos & Fenol & 2,715 & 81 \\
\cline { 2 - 5 } & Alcoholes & Metanol & 0,687 & 90 \\
\cline { 2 - 5 } & $\begin{array}{c}\text { Ácidos } \\
\text { Carboxílicos }\end{array}$ & Ácido Acético & 0,806 & 91 \\
\cline { 2 - 5 } & Inorgánicos & Azufre (S8) & 17,430 & 88 \\
\cline { 2 - 5 } & Nitrogenado & Octahidroindolizina & 1,998 & - \\
\hline \multirow{5}{*}{ Fotoquímica(4) } & Carbonilo & 2,5-Hexanodiona & 1,731 & 91 \\
\cline { 2 - 5 } & Ácidos & Ácido propanoico & 0,918 & 86 \\
\cline { 2 - 5 } & Carboxílicos & Ácido Acético & 0,812 & 94 \\
\cline { 2 - 5 } & Nitrogenado & Octahidroindolizina & 1,997 & - \\
\hline
\end{tabular}

Tabla 6: Determinación de compuestos orgánicos en mezclas complejas por GC/MS para muestras de oxidación avanzada fotoquímica y no fotoquímica.

En la Tabla 6 se observa que las reacciones fotoquímicas fueron eficientes en la oxidación de las especies orgánicas a carbonilo y a ácidos carboxílicos, logrando de esta manera transformar las especies presentes en la muestra en materia orgánica degradable y minerarizable para el tiempo de reacción diseñado (Vieira et al. 2016; Poyatos et al. 2010). Adicionalmente se pudo observar en la Tabla 6 que hay una especie que comparte las tres reacciones, con 
respecto a las especies nitrogenadas presentes en las muestras. Éste compuesto es la octahidroindolizina ( $\gamma$-coniceina), el cual pudo haberse formado a partir de la oxidación de una especie orgánica nitrogenada (aminocetona) mediante la vía de fotociclización reductiva (Hjelmgaard et al. 2007). Otra ruta para el cierre del anillo de la aminocetona es mediante la técnica metátesis de cierre de anillo carbonilo-olefina catalizada mediante cloruro férrico u oxido de titanio en presencia de 1,2-dicloroetano a bajas temperaturas (temperatura alcanzada durante la refrigeración de la muestra), pudiendo alcanzar condiciones para formar el compuesto (Ma et al. 2016).

\section{Caracterización del catalizador original, gastado y solidos generados durante la fotoreacción}

Los resultados obtenidos del análisis de difracción de rayos $\mathrm{X}$ para las muestras del catalizador original (línea inferior color rojo) se observan en la Figura 4, notándose que las estructuras cristalinas predominantes son el cuarzo, hematita, bohemita y la anatasa, también se identificó la presencia de otras especies como la cancrinita tipo sodalita (Arteaga, 2015; Saputra et al. 2012). Entre las principales especies cristalinas identificadas para el lodo rojo generado por CVG Bauxilum neutralizado y calcinado a $400{ }^{\circ} \mathrm{C}$ (LRS400, línea inferior de color rojo ), se encuentra el cuarzo $\mathrm{SiO}_{2}$ (COD: 01-087-2096), hematita $\alpha-\mathrm{Fe}_{2} \mathrm{O}_{3}$ (COD: 01076-4579 ), anatasa $\mathrm{TiO}_{2}(\mathrm{COD}: 01-075-2547)$, cancrinita $\mathrm{Na}_{8}\left(\mathrm{Al}_{6} \mathrm{Si}_{6} \mathrm{O}_{24}\right)\left(\mathrm{CO}_{3}\right)\left(3 \mathrm{H}_{3} \mathrm{O}\right)$ (COD: 01089-9099) y boehmita a-AlO(OH) (COD: 01-073-9095). Se puede observar que las especies que presentaron mayor señal en el difractograma fueron aquellas que contienen aluminio en su estructura cristalina como la boehmita, evidenciando el contenido de aluminio en las muestras estudiadas, otro de los componentes mayoritarios de los lodos rojos es la hematita $\left(\mathrm{Fe}_{2} \mathrm{O}_{3}\right)$, (Centeno-Bordones et al. 2020).

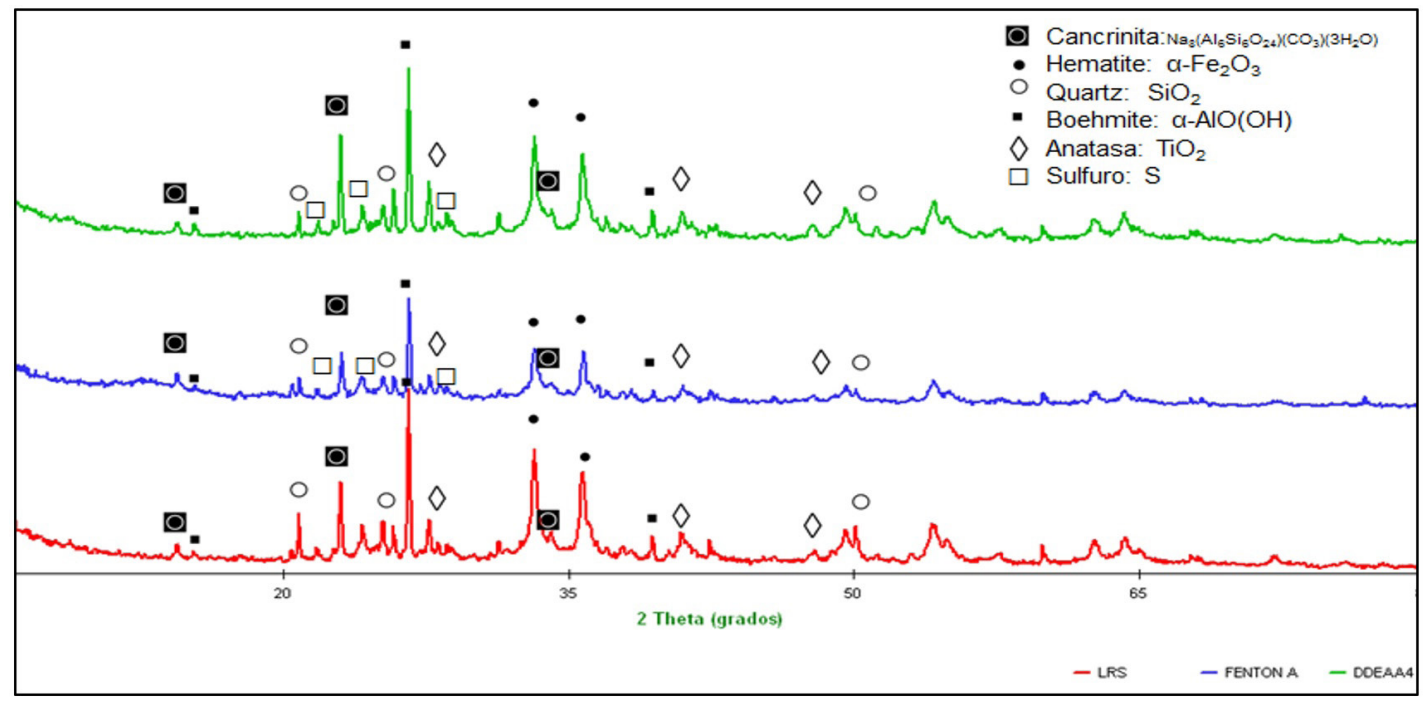

Fig. 4: Composición mineralógica mediante difracción de rayos $X$ del catalizador lodo rojo LRS400 (difractograma inferior); Fenton-Like, (difractograma central) se refiere al catalizador lodo rojo gastado mediante la técnica no fotoquímica (Fenton-Like 4); Oxidación Fotoquímica (difractograma superior) catalizador gastado en el proceso de oxidación avanzada utilizando radiación solar ( FQ4). 
En la Figura 4, se observa en difractograma central (color azul) el catalizador gastado mediante la técnica de oxidación Fenton-like, y en difractograma superior (color verde) que las especies cristalinas presentes en el catalizador no sufren ninguna modificación debido a las condiciones de los POA sin radiación y con radiación solar, lo que sí se evidencia en estos materiales gastados son las señales correspondiente al Sulfuro (COD: 04-012-7311). Esto podría deberse a que una parte del contenido de $\mathrm{H}_{2} \mathrm{~S}$ disuelto que contiene el agua agria del mejoramiento de crudos venezolanos, es sorbido por el catalizador debido a sus características químicas de alta reactividad y afinidad electrónica a un $\mathrm{pH}$ 2, cuando la densidad de cargas positivas es mayor, permitiendo la afinidad con especies cargadas negativamente a este $\mathrm{pH}$ como los sulfuros en el medio acuoso (Araujo y Vázquez, 2015). La remoción del sulfuro de hidrógeno $\left(\mathrm{H}_{2} \mathrm{~S}\right)$ puede ser a través de la adsorción, donde los materiales utilizados deben contener óxidos, óxidos hidratados o hidróxido de hierro. Los materiales utilizados fueron el $\mathrm{Fe}_{2} \mathrm{O}_{3}$ y el hidróxido de hierro $\mathrm{Fe}(\mathrm{OH})_{3}$ en la superficie hidratada del catalizador, los cuales capturan el sulfuro de hidrógeno, formando sulfuro de hierro y agua, ecuaciones (6) y (7). Las reacciones de formación del sulfuro de hierro para el óxido son ligeramente endotérmicas, por lo que la temperatura del ambiente es suficiente para hacer posible la reacción de desulfuración (Torres-Calderón et al. 2020).

$$
\begin{aligned}
& \mathrm{Fe}_{2} \mathrm{O}_{3}+3 \mathrm{H}_{2} \rightarrow \mathrm{Fe}_{2} \mathrm{O}_{3}+3 \mathrm{H}_{2} \mathrm{O} \\
& 2 \mathrm{Fe}(\mathrm{OH})_{3}+3 \mathrm{H}_{2} \mathrm{~S} \rightarrow \mathrm{Fe}_{2} \mathrm{~S}_{3}+6 \mathrm{H}_{2} \mathrm{O}
\end{aligned}
$$

Durante el proceso de neutralización del agua agria tratada por POA fotoquímico se genera un sólido vitro-brilloso de color pardo, identificado como óxido de hierro trihidratado $\left(2 \mathrm{Fe}_{2} \mathrm{O}_{3} \cdot 3 \mathrm{H}_{2} \mathrm{O}\right)$ en fase amorfa (García-Murillo 1994). Este material se forma debido a la lixiviación del hierro del catalizador a $\mathrm{pH}$ 2, conduciendo a una acumulación de Fe en la solución. $\mathrm{El} \mathrm{Fe}{ }^{+3}$ comienza a precipitar en el proceso de neutralización, cuando el $\mathrm{pH}$ es cercano a 3, formando oxihidróxido de hierro (Zhang et al. 2019; Salgado et al. 2013).

La formación del lodo férrico ocurre como resultado del proceso de hidroxilación de la hematita (grupo espacial romboédrico) que en proceso de hidratación se produce la formación de los grupos $\mathrm{OH}$ en la estructura y su posterior transformación estructural a la fase goethita (grupo espacial ortorrómbico). Las Ecuaciones (8) y (9) describen la hidroxilación de la fase cristalina goethita y la fase amorfa limonita, respectivamente (Palacios et al. 2012).

$$
\begin{aligned}
& \alpha \mathrm{Fe}_{2} \mathrm{O}_{3}+\mathrm{H}_{2} \mathrm{O} \rightarrow 2 \alpha \mathrm{FeO}(\mathrm{OH}) \\
& \alpha \mathrm{FeO}(\mathrm{OH})+n \mathrm{H}_{2} \mathrm{O} \rightarrow \alpha \mathrm{FeO}(\mathrm{OH}) . n \mathrm{H}_{2} \mathrm{O}
\end{aligned}
$$

Una de las ventajas que tienen los POA fotoquímicos de tipo heterogéneo, es la baja lixiviación del hierro, afectando lo menos posible el ciclo fotoredox $\mathrm{Fe}^{+3} \leftrightarrow \mathrm{Fe}^{+2}$. Presentando una baja producción de limonita, permitiendo la eficiencia en la generación de radicales libres para la oxidación de los contaminantes orgánicos e inorgánicos, garantizando la estabilidad del catalizador y su reutilización (Zhang et al. 2019; Ganiyu et al. 2018).

En la Figura 5 se presentan los espectros infrarrojos de los sólidos estudiados, El espectro de color negro (segunda espectro descendente) representa el catalizador original de lodo 
rojo utilizado (LRS400), coincidiendo con las bandas reportadas por Centeno-Bordones et al. (2020), En el espectro se aprecian las bandas correspondientes a la hematita entre 470 y $540 \mathrm{~cm}^{-1}$, correspondientes a las observadas a 560 y $460 \mathrm{~cm}^{-1}$ según Arteaga 2015; López y Linares, 2018. En la región inferior a $1000 \mathrm{~cm}^{-1}$, se observan bandas correspondientes a vibraciones de tensión de Si-Al-O, posiblemente asociados a la cancrinita presente como fase cristalina detectada en el lodo rojo, estas señales tienden a solapar las señales características del enlace O-Ti-O (Arteaga 2015; López y Linares, 2018). Las bandas observadas cerca de 1406 y $1483 \mathrm{~cm}^{-1}$ se deben a las vibraciones del ion carbonato (Liu et al. 2011). Mientras que las bandas que aparecen a 1585 y $1600 \mathrm{~cm}-1$ se deben a las vibraciones de flexión de la molécula de agua (Jankovic et al. 2013; Villacís-García et al. 2015; Arteaga 2015; López y Linares, 2018; Centeno-Bordones et al. 2020). En todos los espectros se presentan unas bandas en el intervalo entre 3000 y $3400 \mathrm{~cm}^{-1}$, las cuales pueden atribuirse a los diferentes grupos $\mathrm{OH}$ solapados, en especial aquellos asociados al aluminio, así como al agua de hidratación de la cancrinita (Jankovic et al. 2013; Villacís-García et al. 2015; Arteaga 2015; López y Linares, 2018; Centeno-Bordones et al. 2020).

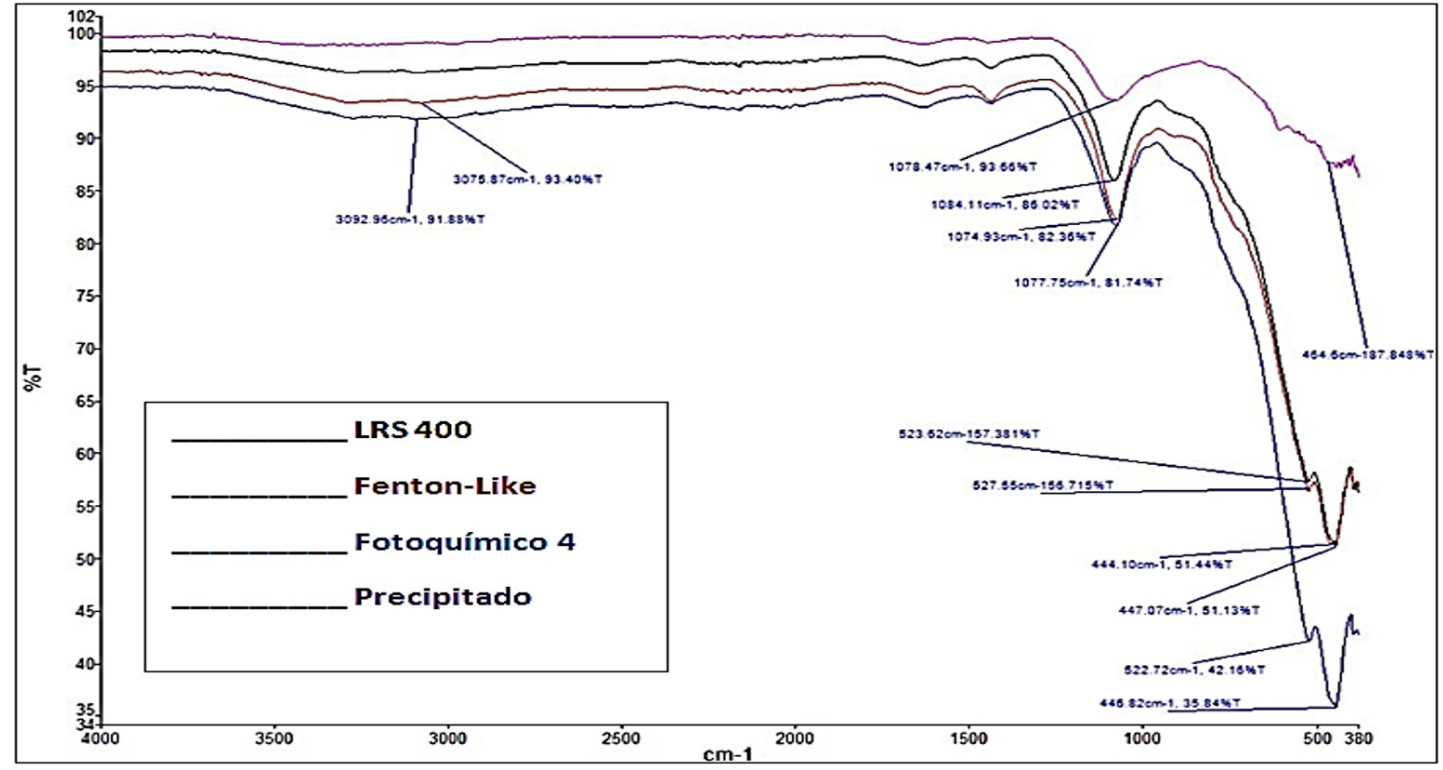

Fig. 5: Espectro infrarrojo con transformada de Fourier para los sólidos que intervienen en el proceso de oxidación avanzada. El segundo espectro descendente corresponde al catalizador LRS400

original, el tercer espectro descendente se refiere al catalizador gastado mediante el proceso FentonLike, el cuarto espectro se refiere al catalizador gastado en el proceso fotoquímico de oxidación y el primer espectro superior se refiere al sólido precipitado en el proceso de neutralización.

En los espectros del sólido gastado en la reacción no fotoquímica de tipo Fenton-like de color rojo (tercer espectro descendente) y del sólido del proceso fotoquímico de color azul (cuarto espectro descendente), se puede apreciar que en el infrarrojo lejano por debajo de $400 \mathrm{~cm}^{-1}$ se observan algunas bandas que pudiesen ser típicas del enlace Fe-S (Inaba y Kimura, 2004). De la misma manera en los límites del infrarrojo cercano con señales entre 444 y $527 \mathrm{~cm}^{-1}$, pudiéndose éstas referirse al enlace $\mathrm{Fe}-\mathrm{O}$ típicos de la hematita. En esta zona 
se casi en solapamiento las señales correspondientes a las especies $\mathrm{M}_{\mathrm{X}} \mathrm{SO}_{4}$ entre 400 y 500 $\mathrm{cm}^{-1}$, tales como la tenardita $\left(\mathrm{Na}_{2} \mathrm{SO}_{4}\right)$, anhidrita $\left(\mathrm{CaSO}_{4}\right)$, arcanita $\left(\mathrm{K}_{2} \mathrm{SO}_{4}\right)$, yeso hemihidratado $\left(\mathrm{CaSO}_{4} \cdot 1 / 2 \mathrm{H}_{2} \mathrm{O}\right.$ ), yeso dihidratado $\left(\mathrm{CaSO}_{4} \cdot 2 \mathrm{H}_{2} \mathrm{O}\right.$ ), (Vásquez- Moreno y Blanco- Valera, 1981; García- Murillo, 1994), lo cual explicaría que las líneas anteriores se expresen intensas para Fenton-Like, pero mucho más intensas y notorias para el proceso fotoquímico.

Luego se distingue la señal correspondiente a 1074 y $1077 \mathrm{~cm}^{-1}$, que pudiese corresponder a los enlaces típicos de Si-Al-O, a la tensión entre O-Ti-O, al estiramiento simétrico del enlace de sulfuros S-O-R y sulfóxidos S-O, así como también del ion $\mathrm{SO}_{4}{ }^{-2}$ (Silverstein et al. 2005). En este espectro también se ve una tenue señal alrededor de $10 s 1400 \mathrm{~cm}^{-1}$ banda correspondiente al ion carbonato, o al enlace C-O (Silverstein et al. 2005). De la misma manera en el orden de los $1600 \mathrm{~cm}^{-1}$ se observa una señal típica de las vibraciones de flexión del agua, que puede proceder de la hidratación de algunos de los compuestos del catalizador gastado, al mismo tempo esta señal es confirmada con una señal a 3075 y $3092 \mathrm{~cm}^{-1}$ típica de las bandas del $\mathrm{OH}$ y de agua.

Con respecto al espectro del precipitado en el proceso de neutralización del proceso de oxidación avanzada solar se pueden apreciar las señales del enlace $\mathrm{Fe}-\mathrm{O}$ alrededor de 454 $\mathrm{cm}^{-1}$. También se observa una señal intensa y ancha en la banda del $1078 \mathrm{~cm}^{-1}$, que pudiese corresponder a los enlaces típicos de sulfóxidos $\mathrm{S}-\mathrm{O}$, sulfuros $\mathrm{S}-\mathrm{O}-\mathrm{R}$ y sulfatos, estos últimos que pudiesen precipitar debido al cambio de $\mathrm{pH}$ en la solución (Castillo-López y ChávezPozo, 2016). Por último se nota una leve señal cercana al $1600 \mathrm{~cm}^{-1}$ que pudiese deberse a la flexión del agua en el material y una leve señal en el orden de los $3000 \mathrm{~cm}^{-1}$ típica de los enlaces $\mathrm{OH}$ presentes en el oxihidróxido de hierro.

\section{CONCLUSIONES}

La aplicación de lodos rojos activados en los procesos de oxidación avanzada utilizando radiación solar para el tratamiento de aguas agrias provenientes del proceso de mejoramiento de crudos pesados y extrapesados, obtuvo una alta eficiencia en la oxidación de compuestos orgánicos presentes en este efluente industrial, llevándolos a la degradación y mineralización total. Adicionalmente, el tratamiento permitió la oxidación de los compuestos inorgánicos, principalmente al eliminar los sulfuros del medio acuoso y convertirlos en sulfatos con un rendimiento del 99,99\%, esto debido a la presencia de manganeso en el catalizador y su reacción con ácido sulfúrico, lo que permitió la transformación del $\mathrm{H}_{2} \mathrm{~S}$ a sulfato por la acción catalítica del sulfato de manganeso.

El diseño de experimento fotoquímico (fotoFenton-like y fotocatálisis), obtuvo excelentes rendimientos en la oxidación de la materia orgánica presente en el efluente, llegando a obtener en la mejor corrida experimental una reducción de la DQO en un 88,95 \% y un $100 \%$ de reducción de TPH. El análisis de varianza determinó que las variables cantidad de catalizador y cantidad de peróxido son significativas en la reducción de la DQO, y en el análisis de la superficie de respuesta se obtuvo que los niveles experimentales $(1,1)$ fueron los óptimos para la disminución de la materia orgánica en el agua agria. Siendo las condiciones experimentales optimas $6 \mathrm{~g} / \mathrm{L}$ de catalizador, $100 \mathrm{~g} / \mathrm{L}$ de peróxido de hidrógeno al 30\%, con un ajuste a pH 2 y en tiempo de reacción de 4 h.

La interacción entre el hierro del catalizador y la molécula del peróxido se incrementa a 
medida que se acidifica el medio de reacción, permitiendo el acercamiento entre los sitios activos del catalizador y el peróxido para la producción de radicales oxidrilo. La disminución del pH tiene influencia en la lixiviación del hierro debido a que incrementa su concentración en el efluente tratado, obteniéndose un porcentaje de lixiviación de hierro de 3,26\% demostrando que la actividad de la reacción se lleva a cabo vía catálisis heterogénea en un 96,84\%. Lo anterior se confirma en los estudios de sorción de carbono donde se comprobó que bajo las condiciones experimentales la reducción de la DQO y el TPH se debe en un $99,86 \%$ a procesos catalíticos desarrollados por el lodo rojo activado.

Las especies orgánicas identificadas en la reacción son las de mayor estado de oxidación orgánica, logrando el diseño experimental propuesto obtener especies del grupo carbonilo y ácidos carboxílicos de cadena corta, siendo estos compuestos de menor impacto ambiental, los cuales podrían ser asimilados y metabolizados por microorganismos en las plantas de tratamiento de aguas residuales. 


\section{REFERENCIAS}

Alboudwarej, H., Felix, J., Taylor, S., Badry, R., Bremner, C., Brough, B., Beshry, M. (2006). La importancia del petróleo pesado. Oilfield review, 18(2), 38-58. Obtenido de: https:// d1wqtxts1xzle7.cloudfront.net/41075647/CRUDOS_PESADOS.pdf?1452693971=\&responsecontent-disposition=inline \%3B+filename\%3DMaquetacion_1.pdf

Araujo, A. G. T., Vázquez, J. A. R. (2015). Técnicas para la disminución en la concentración de ácido sulfhídrico en el biogás. Jóvenes en la Ciencia, 1(2), 1449-1453. Obtenido de: http:// www.jovenesenlaciencia.ugto.mx/index.php/jovenesenlaciencia/article/view/74

Arteaga (2015) Evaluación de catalizadores Mo/lodos rojos modificado empleando diseño de experimento para el hidrotratamiento de corrientes LCO. Trabajo especial de grado. Universidad de Carabobo.

ASTM D 4658-03 (2002) Standard Test Method for Sulfide Ion in Water.

ASTM D5790-95 (2012) Standard Test Method for Measurement of Purgeable Organic Compounds in Water by Capillary Column Gas Chromatography/Mass Spectrometry

Bhatnagar, A., Vilar, V. J., Botelho, C. M., Boaventura, R. A. (2011). A review of the use of red mud as adsorbent for the removal of toxic pollutants from water and wastewater. Environmental technology, 32(3), 231-249. https://doi.org/10.1080/09593330.2011.560615

Castillo López, V. M., Chávez Pozo, R. R. (2016). Influencia del pH y dosis de hidróxido de aluminio sobre el porcentaje de eliminación de sulfatos en agua de mina artificial mediante tratamiento por precipitación mineral. Obtenido de: http://dspace.unitru.edu.pe/ handle/UNITRU/2552

Castro, Y., Sánchez, D., Viloria, A. (2019). Efecto de composición mineral de arenas sobre generación de gases ácidos en crudos pesados a condiciones de inyección de vapor. Revista INGENIERÍA UC, 26(1), 23-30. Obtenido de: https://www.redalyc.org/ jatsRepo/707/70758484004/70758484004.pdf

Centeno-Bordones G, Jimenez, Y. (2018). Uso de Lodos Rojos como catalizador en los procesos de oxidación avanzada: una aproximación al estado del arte. Tekhné, 21(4). Obtenido de: http://revistasenlinea.saber.ucab.edu.ve/temas/index.php/tekhne/article/ view/3940

Centeno-Bordones, G., Jiménez, Y., y García, J. V. (2020). Caracterización fisicoquímica de los lodos rojos neutralizados y calcinados con posible actividad catalítica en procesos de oxidación avanzada. Tekhné, 23(1). Obtenido de : http://revistasenlinea.saber.ucab.edu.ve/ temas/index.php/tekhne/article/view/4494

Centro Nacional de Tecnología Química (2012) Informe de prensa: Gestión CNTQ 2012. Obtenido de: www. Cienciasguayana.com/2012/01/fonacit-destina-recursos-para.html.

Clemente, J (2010) aplicación de procesos de oxidación avanzada (fotocatálisis solar) para tratramientos y reutilización de efluentes texiles. Tesis doctoral, Universidad Politécnica de Valencia, España. Obtenido de: https://dialnet.unirioja.es/servlet/dctes?codigo=18128

Folgoso, J (2015) Utilización de lodos rojos como absorbentes. Tesis doctoral. Universidade da Coruña. España. Obtenido de: https://ruc.udc.es/dspace/handle/2183/14828

Gaceta Oficial 52453 (1998). Decreto 2635: Normas para el control y manejo de materiales 
y desechos peligrosos. Caracas, Republica de Venezuela. Obtenido de: http://ley.tuabogado. com/leyes/normas/decreto-2635-normas-para-el-control-y-manejo-de-materiales-ydesechos-peligrosos-gaceta-5245-1998\#gsc.tab=0

Galarraga (2002) CVG-Bauxilum red mud neutralization.Journal the materials, metals \& metal society, 23, 110-134. Obtenido de: https://jglobal.jst.go.jp/en/detail?JGLOBAL_ ID $=200902126325207529$

Ganiyu, S. O., Zhou, M., Martínez-Huitle, C. A. (2018). Heterogeneous electro-Fenton and photoelectro-Fenton processes: a critical review of fundamental principles and application for water/wastewater treatment. Applied Catalysis B: Environmental, 235, 103-129. https:// doi.org/10.1016/j.apcatb.2018.04.044

Garcés-Giraldo, L. F., Mejía-Franco, E. A.,Santamaría-Arango, J. J. (2004). La fotocatálisis como alternativa para el tratamiento de aguas residuales.Obtenido de: http://repository. lasallista.edu.co/dspace/handle/10567/195

García- Murillo, M A (1994). Espectroscopia infrarroja de minerales. Instituto Politécnico Nacional. Ciudad de México, México. Obtenido de: https://tesis.ipn.mx/jspui/ bitstream/123456789/16863/1/25-1-12809.pdf

González, J. L., Núñez, C. R. (2008). Mejoramiento de los crudos extra pesados Carabobo y Ayacucho mediante hidrotratamiento utilizando catalizadores CoMoS/y-Al2O3 y FeNiNbS másicos en un reactor por carga. (Tesis de Licenciatura), Universidad de Carabobo, Venezuela.Obtenido de: http://www.riuc.bc.uc.edu.ve/handle/123456789/7326

Guimarães, J. R., Gasparini, M. C., Maniero, M. G., y Mendes, C. G. (2012). Stripped sour water treatment by advanced oxidation processes. Journal of the Brazilian Chemical Society, 23(9), 1680-1687.

http://dx.doi.org/10.1590/S0103-50532012005000031

Gupta, V. K., Ali, I., Suhas, y Mohan, D. (2003). Equilibrium uptake and sorptiondynamics for the removal of a basic dye (basic red) using low-cost adsorbents. Journal of Colloid and Interface Science, 265, 257-264. https://doi.org/10.1016/S0021-9797(03)00467-3

Gutierrez y De la Vara (2008) Analisis y diseño de experimentos, Segunda edicion. Editorial Mc Graw Hill. Mexico.

Hinojosa, O. (2002). Oxidación de sulfuros: importante proceso de pretratamiento.

Revista Metalúrgica UTO, 31. Obtenido de: http://www.revistasbolivianas.org.bo/scielo. php?pid=S2078-55932002000100006\&script=sci_abstract

Hjelmgaard, T., Gardette, D., Tanner, D., Aitken, D. J. (2007). Synthesis of (+)-coniceine via reductive photocyclization of dienamides: an entry to indolizidines. Tetrahedron: Asymmetry, 18(5), 671-678. https://doi.org/10.1016/j.tetasy.2007.03.004

Inaba, S., Kimura, Y. (2004). Behavior of sulfur in the carbon-bearing iron oxide pellet during heating. ISIJ international, 44(12), 2112-2114. https://doi.org/10.2355/ isijinternational.44.2112

Jang, J. H., Dempsey, B. A., Burgos, W. D. (2007). Solubility of hematite revisited: Effects of hydration. Environmental science \& technology, 41(21), 7303-7308. https://doi.org/10.1021/ es070535t 
Jankovic, B., Smiciklas, I., Stajic-Trosic, J., Antonovic, D. (2013). Thermal characterization and kinetic analysis of non-isothermal decomposition process of Bauxite red mud. Estimation of density distribution function of the apparent activation energy. International Journal of Mineral Processing, 123, 46-59. https://doi.org/10.1016/j.minpro.2013.05.003

Liu, D. Y., Wu, C. S. (2012). Stockpiling and comprehensive utilization of red mud research progress. Materials, 5(7), 1232-1246. https://doi.org/10.3390/ma5071232

Lin, S. S., Gurol, M. D. (1998). Catalytic decomposition of hydrogen peroxide on iron oxide: kinetics, mechanism, and implications. Environmental Science \& Technology, 32(10), 14171423. https://doi.org/10.1021/es970648k

Liu, Q., Xin, R., Li, C., Xu, C., Yang, J. (2013).Application of red mud as a basic catalyst for biodiesel production. Journal of Environmental Sciences, 25(4), 823-829. https://doi. org/10.1016/S1001-0742(12)60067-9

Liu, X., Zhang, N., Sun, H., Zhang, J., Li, L. (2011). Structural investigation relating to the cementitious activity of bauxite residue-Red mud. Cement and Concrete Research, 41(8), 847-853. https://doi.org/10.1016/j.cemconres.2011.04.004

López, D., Linares, C. (2018). Characterization of the red mud calcined to different temperatures. INGENIERIA UC, 25(1), 86-92. Obtenido de : http://servicio.bc.uc.edu.ve/ ingenieria/revista/v25n1/art10.pdf

Ma, L., Li, W., Xi, H., Bai, X., Ma, E., Yan, X., Li, Z. (2016). FeCl3-Catalyzed Ring-Closing Carbonyl-Olefin Metathesis. Angewandte Chemie International Edition, 55(35), 1041010413. https://doi.org/10.1002/anie.201604349

Marquez, J. J. R. (2015). Viabilidad de la fotólisis del peróxido de hidrógeno (H2O2/UV) para la reutilización de aguas residuales industriales: aplicación a las aguas de una refinería de petróleo (Doctoral dissertation, Universidad de Cádiz). Obtenido de: https://dialnet. unirioja.es/servlet/tesis? codigo $=51119$

Martínez, F., Calleja, G., Melero, J.A., Molina, R. (2004). Heterogeneous photo-Fenton degradation of phenolic aqueous solutions over iron-containing SBA-15 catalyst. Applied Catalysis B: Environmental, 60:181-190.https://doi.org/10.1016/j.apcatb.2005.03.004

Montes, M. V. (2014). Assessment of polychlorinateddibenzo-p-dioxins and dibenzofurans, PCDD/Fs, in the application of advanced oxidation processes (Doctoral dissertation, Universidad de Cantabria).Obtenido de : https://dialnet.unirioja.es/servlet/ tesis? codigo $=43831$

Noguera, G., Lara, M., (2013) Uso de aguas agrias y H2S para la preparación de soluciones metálicas de [MoS4]2-. [Informe Técnico]. Los Teques, Venezuela: Intevep. Documento técnico n: INT- 14214,2013.

Noguera, G., Rivas, A., González, I., Hernandez, J., (2012) Evaluación del uso de aguas agrias en la preparación de solución de Mo(VI) de catalizador ultradisperso de HDHPLUS ${ }^{\circledR}$. [Informe Técnico]. Los Teques, Venezuela: Intevep,. Documento técnico nº INT- 14040, 2012.

Palacios, P. R., De Los Santos Valladares, L., Bustamante, A., González, J. C. (2012). Estudio de la deshidroxilación en el óxido férrico hidratado denominado limonita. Revista de la Sociedad Química del Perú, 78(3), 198-207. Obtenido de: http://www.scielo.org.pe/scielo. 
php?pid=S1810-634X2012000300006\&script=sci_arttext

Pereira, M., Oliveira, L., Murad, E (2012), Iron oxide catalysts: Fenton and Fentonlike reactions: a review, Clay Minerals 47, 285-302. https://doi.org/10.1180/ claymin.2012.047.3.01

Poyatos, J. M., Muñio, M. M., Almecija, M. C., Torres, J. C., Hontoria, E., Osorio, F. (2010). Advanced oxidation processes for wastewater treatment: state of the art. Water, Air, and SoilPollution, 205(1-4), 187. Obtenido de: https://link.springer.com/article/10.1007/s11270009-0065-1

Ramos, K (2017) Evaluación de procesos de oxidación avanzados: foto-fenton con luz solar para la degradación de sustancias y desechos líquidos tóxicos. (Tesis Doctoral), Universidad de Carabobo. Valencia, Venezuela. Obtenido de: http://mriuc.bc.uc.edu.ve/ handle/123456789/7203

Ramos, K., Jiménez, Y. (2016). Aplicación de diseño de experimentos en la degradación de desechos por el proceso foto-Fenton heterogéneo usando un óxido mixto de MgFeAl. Saber, 28(3): 574-582. Obtenido de: https://www.redalyc.org/ jatsRepo/4277/427750771014/427750771014.pdf

Rios-Enriquez, M. A., Bossmann, S. H., Oliveros, E., Shahin, N., Braun, A. M., Durande-Bazua, C. (2003). A"well-known"advanced oxidation reaction revisited. The photoFenton-oxidation of 4-chlorophenol and 2, 4-dichloro-phenol in a homogeneous and a heterogeneous system. Obtenido de: https://www.osti.gov/etdeweb/biblio/20475882

Rubio-Clemente, A., Chica, E. L., Peñuela, G. A. (2014). Aplicación del proceso Fenton en el tratamiento de aguas residuales de origen petroquímico. Ingeniería y competitividad, 16(2), 211-223. Obtenido de: https://revistaingenieria.univalle.edu.co/

Salas, G. (2005). Eliminación de sulfuros por oxidación en el tratamiento del agua residual de una curtiembre. Revista peruana de Química e ingeniería química, 8(1), 49-54. Obtenido de: https://revistasinvestigacion.unmsm.edu.pe/index.php/quim/article/view/5152/0

Salgado, P., Melin, V., Contreras, D., Moreno, Y., Mansilla, H. D. (2013). Fenton reaction driven by iron ligands. Journal of the Chilean Chemical Society, 58(4), 2096-2101. http:// dx.doi.org/10.4067/S0717-97072013000400043

Saputra, E., Muhammad, S., Sun, H., Ang, H. M., Tadé, M. O., Wang, S. (2012). Red mud and fly ash supported Co catalysts for phenol oxidation. Catalysis Today, 190(1), 68-72. https://doi.org/10.1016/j.cattod.2011.10.025

Silverstein, R. M., Webster, F., Kiemle D.(2005). Spectrometric identification of organic compounds. Seventh edition . Jhon Wiley and son, inc. New York, USA.

Standard Methods for Examination of Water and Wastewater 4500-Cl- B. (2017). Chlorine, Iodometric method.

Torres-Calderón, S., Paucar-Palomino, M. J., Pampa-Quispe, N. B. (2020). Adsorción de sulfuro de hidrógeno del biogás mediante virutas de hierro pretratadas para su reaprovechamiento energético. Revista de Ingeniería Hidráulica y Ambiental, 41(1), 18-29. https://doi.org/10.1016/j.cattod.2011.10.025

U.S. EPA. 2014. "Method 6020B (SW-846): Inductively Coupled Plasma-Mass Spectrometry," 
Revision 2. Washington, USA.

U.S. EPA Method 8015B. (2000). Total petroleum hydrocarbons (TPH) analysis gasoline and diesel fuel.

U.S. EPA 375.4 (1978) Sulfate (Turbidimetric). method determines sulfate in drinking and surface waters; domestic and industrial wastes. Approved for NPDES.

Vásquez- Moreno., Blanco- Valera (1981) Tabla de frecuencias y espectros de absorción infrarroja de compuestos relacionados con la química del cemento. Consejo Superior de Investigaciones Científicas Licencia Creative Commons 3.0. España. Obtenido de: http:// materconstrucc.revistas.csic.es/index.php/materconstrucc/article/view/1007/0

Vieira R, Gonçalves M y Gomes L (2016) Assessment of the use of red mud as a catalyst for photodegradation of bisphenol A in wastewater treatment, wst 2016309; DOI: 10.2166/ wst.2016.309.

Villacís-García, M., Ugalde-Arzate, M., Vaca-Escobar, K., Villalobos, M., Zanella, R., y Martínez-Villegas, N. (2015). Síntesis en laboratorio de goethita y ferríhidrita de tamaños de partícula controlados. Boletín de la Sociedad Geológica Mexicana, 67(3), 433-446. Obtenido de: http://www.scielo.org.mx/scielo.php?script=sci_arttext\&pid=S1405-33222015000300007

Yamal-Turbay, E., Jaén, E., Graells, M, y Pérez-Moya, M. (2013). Enhanced photo-Fenton process for tetracycline degradation using efficient hydrogen peroxide dosage. Journal of Photochemistry and Photobiology A: Chemistry. 267: 11- 16. https://doi.org/10.1016/j. jphotochem.2013.05.008

Zhang, M. H., Dong, H., Zhao, L., Wang, D. X., y Meng, D. (2019). A review on Fenton process for organic wastewater treatment based on optimization perspective. Science of the total environment. https://doi.org/10.1016/j.scitotenv.2019.03.180 